\title{
Triplet state dynamics in poly(2,5-pyridine diyl)
}

\author{
K.S. Wong ${ }^{\text {a, }}$, Carrie W.Y. Law ${ }^{\mathrm{a}}$, T. Sun ${ }^{\mathrm{a}}$, A.P. Monkman ${ }^{\mathrm{b}}$, M.E. Vaschetto ${ }^{\mathrm{b}}$, \\ L.J. Hartwell ${ }^{\mathrm{b}}$, L.E. Horsburgh ${ }^{\mathrm{b}}$, H.D. Burrows ${ }^{\mathrm{c}}$, M.da G. Miguel ${ }^{\mathrm{c}}$ \\ ${ }^{a}$ Department of Physics, Hong Kong University of Science and Technology, Kowloon, Hong Kong \\ ${ }^{\mathrm{b}}$ OEM group, Department of Physics, University of Durham, Durham, UK \\ ${ }^{\mathrm{c}}$ Department of Chemistry, University of Coimbra, Coimbra, Portugal
}

\begin{abstract}
Pulse radiolysis was used to determine the triplet state energy (2.3 eV) of Poly(2,5-pyridine diyl) (PPY) film which was found to be coincident with the emission energy. Detailed time-resolved photoluminescence (PL) and pump-probe measurements have been applied to study the photoexcited state relaxation dynamics. In films, a very large spectral red-shift $(\sim 0.35 \mathrm{eV})$ for the PL occurred within the first 100 ps whereas no spectral red-shift was observed for the PPY in solution. This result shows clear evidence for the evolution of short-lived singlet emission $\left(S_{1} \rightarrow S_{0}\right)$ at $\sim 450 \mathrm{~nm}$ to long-lived triplet emission $\left(T_{1} \rightarrow S_{0}\right)$ at $\sim 520 \mathrm{~nm}$ for the PPY thin film. Streak camera measurement indicates the long live component has a decay time constant of several ns. The picosecond photo-induced triplet state absorption ( $T_{1} \rightarrow T_{2}$ transition) peaks at $\sim 600 \mathrm{~nm}$ as measured by pump-probe which is consistent with both the radiolysis and cw photoinduced absorption measurements. A triplet lifetime of $\sim 6 \mathrm{~ns}$ is measured which is again consistent with the streak camera measurement. These results lead us to believe that the long live component of the emission from PPY film is in fact phosphorescence. Furthermore, there is evidence that oxygen plays a very important role in the fast triplet radiative lifetime in PPY films. C) 2001 Elsevier Science B.V. All rights reserved.
\end{abstract}

Keywords: Poly(2,5-pyridine diyl); Triplet state energy; PPY films; Time-resolved photoluminescence

Excited states and their relaxation processes in conjugated polymers have been of significant experimental and theoretical interest in the past decade. With the recent demonstration of polymer light emitting diodes (PLED) [1] and near commercialisation of these devices, a better understanding of the excited state properties of conjugated polymers became even more important. Luminescence in PLED is normally via recombination of singlet excitons. Spin statistics dictate that there is a 3:1 probability of triplet exciton compared to singlet exciton formation from electrical injected charge carrier recombination in PLED, thus a polymer in which efficient radiative triplet state recombination can occur will greatly enhance the PLED performance. Indeed a recent realisation of radiative triplet state recombination (i.e. phosphorescence) in organic light emitting device with a quantum efficient as high as 7\% [2] compared to $3-4 \%$ for the singlet exciton emission PLED demonstrated the important role of the triplet state. Therefore, understanding the triplet state manifold and singlet-triplet intersystem crossing for the light emitting polymeric systems are important.

\footnotetext{
${ }^{*}$ Corresponding author. Tel.: +852-2358-7475; fax: +852-2358-1652. E-mail address: phkswong@ust.hk (K.S. Wong).
}

The existence of triplet states in conjugated polymers has been well established [3-6]. The photo-induced triplet state absorption $\left(\mathrm{T}_{1} \rightarrow \mathrm{T}_{2}\right.$ transition) measurements were the most frequent experimental technique to investigate these triplet states, however, direct triplet exciton emission (i.e. $\mathrm{T}_{1} \rightarrow \mathrm{S}_{0}$ ) with microsecond lifetime has been observed in poly(3-hexylthiophene) [7] and ladder-type poly(paraphenylene) [8]. In this paper, we report the measurement of the singlet ground state $\left(\mathrm{S}_{0}\right)$ and first triplet level $\left(\mathrm{T}_{1}\right)$ of Poly(2,5-pyridine diyl) (PPY) using pulse radiolysis. The excited state relaxation dynamics were also investigated using femtosecond time-resolved luminescence and pump-probe techniques. These measurement results lead us to speculate that part of the emission from PPY film is in fact phosphorescence with a lifetime of $\sim 6 \mathrm{~ns}$.

The PPY is a good electron transport material as well as an efficient luminescent polymer, and it has been successfully used in PLED applications [9,10]. However, PPY is somewhat different from many other luminescent conjugated polymers, it has large Stokes shifts between the absorption and emission bands, higher photoluminescence (PL) efficiency in film compared to solution, and is stable in air. Fig. 1 shows the absorption and PL spectra for both solution and film. It is clearly show that the Stokes shift between the 


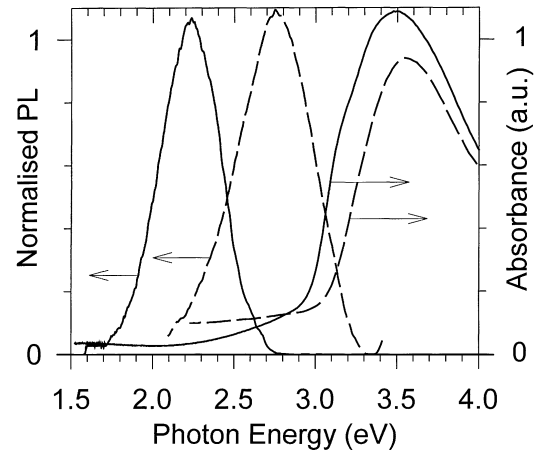

Fig. 1. Absorption and emission spectra of poly(2,5-pyridine diyl) film (solid line) and formic acid solution (dotted line) showing the large shifts in the relative positions of the features and the two states.

absorption and PL peaks is $\sim 1 \mathrm{eV}$ for the PPY film. This has been ascribed to either aggregate formation [11] or excimer formation [12]. In a recent study of the effects of protonation on the photophysics of PPY, we have been able to rule out both of these hypotheses [13] but were unable to unambiguously determine the cause of these large shifts. Further, and more importantly, the photoluminescence quantum yield (PLQY) of PPY behaves very differently from other polymers. The PLQY in the solid state $(0.37)$ is more than double that in (formic acid) solution (0.17). Moreover, in the solid state, the emission is stable in air, but decays rapidly in vacuum, slowly reaching the solution value, although the film absorbances do not alter [14]. This cannot be explained by any previous theory for conjugated polymers.

Pulse radiolysis is a very effective technique to determine the triplet energy and it has been recently applied to determine the $S_{0}-T_{1}$ energy separation for soluble polymers [15]. By applying this pulse radiolysis and energy transfer technique with different energy acceptors, we have also measured the $\mathrm{S}_{0}-\mathrm{T}_{1}$ energy separation for regioregular PPY and find it to be $2.4 \mathrm{eV}$ in benzene solution. Thus, we estimate that with PPY in the solid state, this $\mathrm{S}_{0}-\mathrm{T}_{1}$ energy difference is around $2.3 \mathrm{eV}$. This is just above the energy at which we observe the peak emission from PPY films. Therefore, there is reason to believe that emission in PPY films is possibly predominantly phosphorescence. Furthermore, theoretical calculations of the triplet manifold of PPY [16] yield results in excellent agreement with our radiolysis experiments. Details of the pulse radiolysis measurements of the triplet states in PPY can be found in the paper presented in this conference by Monkman et al. [17].

The above hypothesis is further supported by the femtosecond dynamics of the photoexcited states. Femtosecond time-resolved PL experiments were performed using a regeneratively amplified Ti:Sapphire laser in conjunction with frequency up-conversion; the time resolution of the
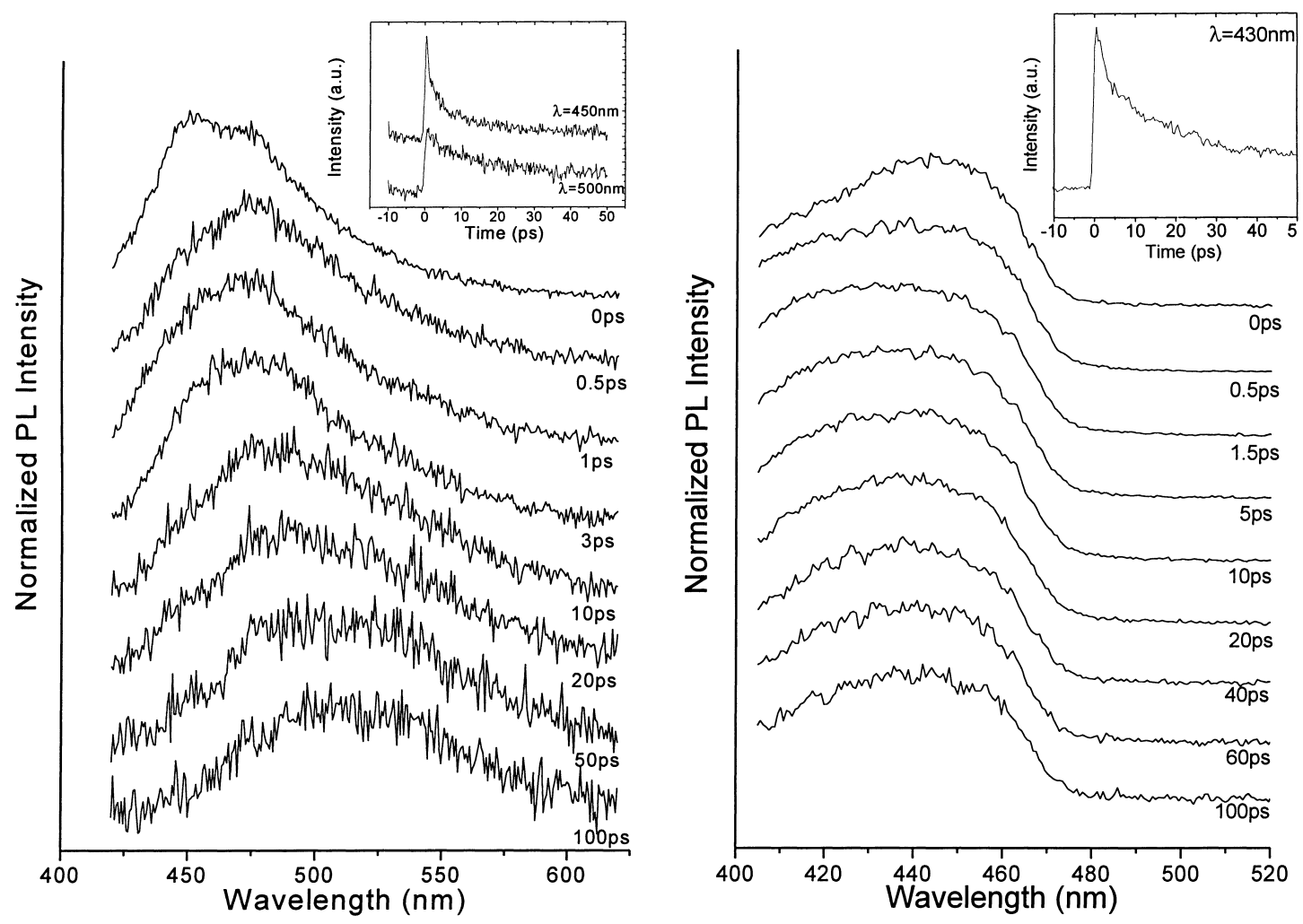

Fig. 2. Spectrally resolved femtosecond time resolved dynamics of the emission from poly(2,5-pyridine diyl) in the solid state. Initial very rapid decay of emission at $450 \mathrm{~nm}$ is observed with the build in of a long-lived component at ca. $525 \mathrm{~nm}$. Inset shows the decay curves measured at both 450 and $500 \mathrm{~nm}$. (b) In the solution state only the rapidly decay emission at $440 \mathrm{~nm}$ is observed, no long lived $525 \mathrm{~nm}$ emission is seen within our time measured time scale (up to $300 \mathrm{ps}$ ). Inset shows the decay curve recorded at $430 \mathrm{~nm}$, unlike the thin film case the decay curves at other wavelengths remain the same. 
system is ca. $300 \mathrm{fs}$ and excitation energy $3.1 \mathrm{eV}$. Time resolved emission spectra from 0 to $100 \mathrm{ps}$ delay of both film and solution are shown in Fig. 2. We observed a rapid and large red-shift of the film PL but no noticeable spectral red-shift for the solution case. In the case of films in air, we observed a very rapidly decay of the solution like emission peak at $450 \mathrm{~nm}$, accompanied by a very long lived species peaking at ca. $525 \mathrm{~nm}$ which is similar to the time-integrated luminescence spectrum (note, films were used for many hours under continuous illumination in the open lab with very little sign of degradation). The figure insets show examples of the PL decay. For film, PL decay is multicomponents and has strong wavelength dependence whereas the PL decay is essentially wavelength independent for the solution case. For PPY thin film at short wavelengths, i.e. $450 \mathrm{~m}$ or less, the fast decay components (few ps to few $10 \mathrm{ps}$ ) dominated, however, a long decay component can still be clearly seen. Measuring emission at $525 \mathrm{~nm}$, we observed mostly the long-lived species with a small contribution from the fast decaying component. We can clearly measure the long lived component by using a streak camera which can extend the time-resolved PL range to several nanoseconds. This measurement verified that the long-lived component has a lifetime of $\sim 5 \mathrm{~ns}$. Thus, we conclude that the very fast signal arises from the radiative decay of singlet states with the long-lived species being due to the phosphorescence. Fig. 3 shows the time-resolved emission peak position as a function of time. The red shift from short delay time emission at $450 \mathrm{~nm}$ to ca. $515 \mathrm{~nm}$ in $100 \mathrm{ps}$, a shift of some $0.35 \mathrm{eV}$ is far too large to be due to spectral diffusion alone [18,19], especially as the PPY is a rigid rod polymer with a polydispersity of 1.7 [20]. Instead, it simply reflects the dominance of the emission band by singlet emission at short times and triplet emission at longer times.

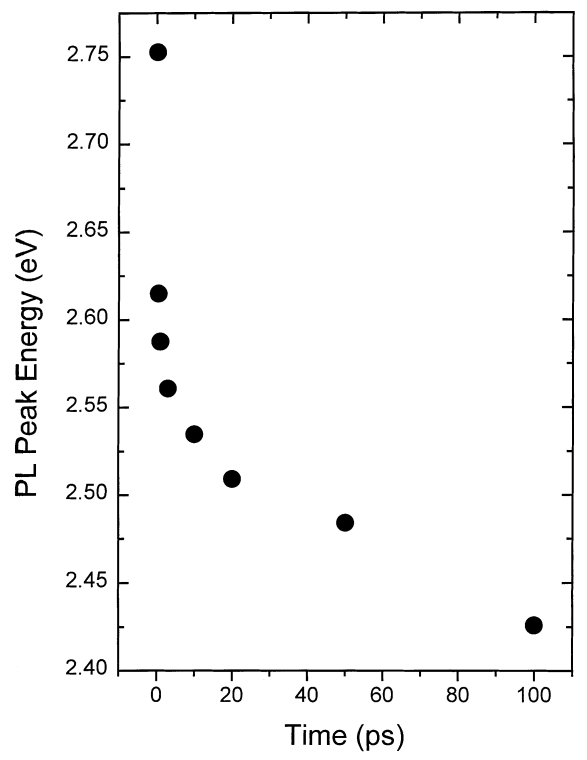

Fig. 3. The change in the emission peak energy as a function of time for the time-resolved PL spectra.

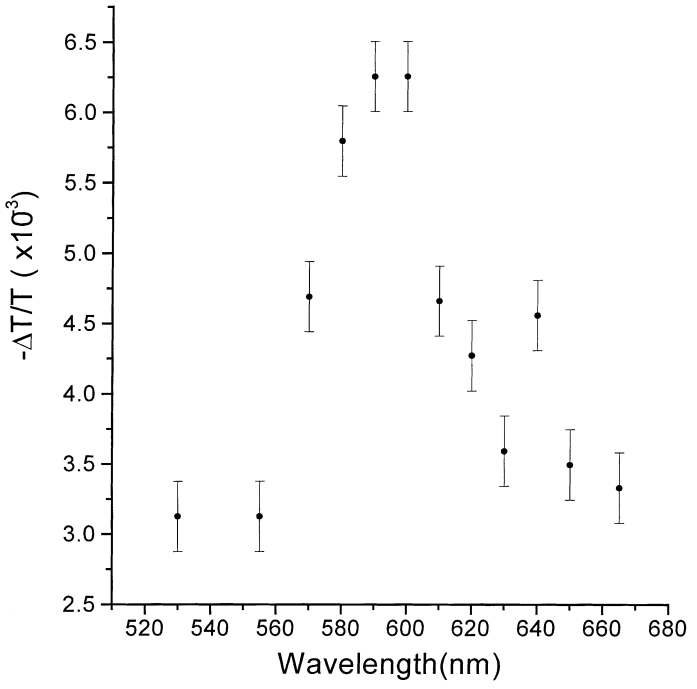

Fig. 4. The photo-induced absorption spectrum of PPY film at $100 \mathrm{ps}$ time delay.

Thus, the very short lifetime of the singlet ( $\tau$ in few ps to few $10 \mathrm{ps}$ ) reflects in part the usual case of spectral diffusion for this type of conjugated polymers and the competition between singlet emission and resonant ISC in PPY.

Fig. 4 shows the picosecond photo-induced absorption (PIA) of the PPY film at $100 \mathrm{ps}$ time delay. The spectrum peak position and shape remain essentially unchanged at other time delays. It has a PIA peak at $\sim 600 \mathrm{~nm}$ which is consistent with the $T_{1}-T_{2}$ absorption peak observed in pulse radiolysis [17]. This result confirms that the picosecond PIA is due to triplet state absorption. Fig. 5 shows the PIA decay at wavelength of $610 \mathrm{~nm}$, a single exponential can be fitted to the decay yielding a lifetime of $\sim 6.5 \mathrm{~ns}$. This triplet state lifetime is consistent with the phosphorescence decay time ( $\sim 5 \mathrm{~ns})$ measured by streak camera. Since a direct generation of triplet excitons by photoexcitation is forbidden, the triplets are normally created by singlet excitons via inter-

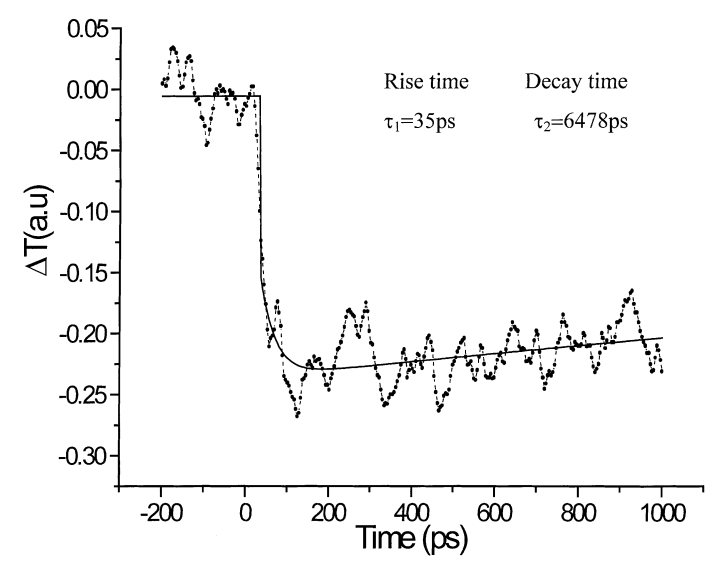

Fig. 5. The photo-induced absorption decay at $\lambda=610 \mathrm{~nm}$. The solid line fitting curve using a exponential rise and decay function with time constant $\tau_{1}$ and $\tau_{2}$, respectively. 
system crossing (ISC) or singlet fission process [8]. The ISC process occurs in $10^{-10}-10^{-9} \mathrm{~s}$ timescale [21], the PIA rise time shown in Fig. 5 is few tens of ps which seems to rule out this process. The singlet exciton fission process would be very fast but it requires the excitation energy to be twice that of the triplet energy [22-24], our present excitation energy at $3.1 \mathrm{eV}$ again make this process unlikely. One possibility of fast triplet generation is by resonant ISC. It is well documented that upon photoexcitation both the singlet [25] and triplet [26] excited states of pyridine have distorted pyridyl ring structures, i.e. a prefulvenic structure [25]. That is the nitrogen atom bending up and out of the plane of the pyridyl ring. This distortion of the ring will enhance mixing of the $n$ and $\pi$ states, increasing the probability of ISC. Density Functional Theory calculations on the PPY system [16] show that the ${ }^{3}\left(n-\pi^{*}\right)$ triplet state is degenerate in energy with the ${ }^{1}\left(\pi-\pi^{*}\right)$, thus this can facilitate resonant (allowed) ISC.

This bring us to the question why triplet state emission appear in PPY film but not in solution, although triplet states are clearly seen in PPY solution by pulse radiolysis measurement. For an explanation, we need again turn to the parent monomer, it has been shown that $\mathrm{O}_{2}$ complexes with the pyridine [27], and that the spin orbit coupling thus introduced perturbs the molecule such that radiative decay of the triplet ground state becomes allowed. Therefore, in polymer films, radiative triplet emission is caused by $\mathrm{O}_{2}$ complexation. However, this complexation must occur in the excited state, i.e. when the chromophore is in the prefulvenic structure, since no direct optical absorption to the triplet state is seen in the polymer. In solution, the two processes will occur to reduce phosphorescence. Protonation does not greatly effect $\left(\pi-\pi^{*}\right)$ transitions whereas it does effect $\left(n-\pi^{*}\right)$ states, strongly shifting the ${ }^{3}\left(n-\pi^{*}\right)$ state out of resonance with the ${ }^{1}\left(\pi-\pi^{*}\right)$ state, such that ISC will be greatly reduced. Also the oxygen complexation site will be blocked on protonation so that the triplet emission is quenched, leaving mainly the singlet emission at $2.6 \mathrm{eV}$. The pulse radiolysis measurements show that in solution the triplets have a very long lifetime, again consistent with the switching off of their radiative decay channel. This also answers the perplexing question as to the instability of PPY emission in vacuum [14]. In air, the relatively triplet lifetime is short (order of 5-6 ns) as show by our picosecond PIA and streak camera measurements, thus there is a much reduced probability of triplet energy transfer to $\mathrm{O}_{2}$ to form singlet oxygen, giving rise to the high stability to photooxidation of the polymer.

In conclusion, pulse radiolysis measurements have determined the triplet state energy of PPY film to be coincident with the emission energy. Detailed time-resolved PL and pump-probe measurements have been applied to study the photoexcited state relaxation dynamics. A very large spectral red-shift $(\sim 0.4 \mathrm{eV})$ for the PL occurred within the first $100 \mathrm{ps}$ for the film case whereas no spectral red-shift was observed for the PPY in solution. This result shows clear evidence for the evolution of short-lived singlet emission $\left(\mathrm{S}_{1} \rightarrow \mathrm{S}_{0}\right)$ at $\sim 450 \mathrm{~nm}$ to long-lived triplet emission $\left(\mathrm{T}_{1} \rightarrow \mathrm{S}_{0}\right)$ at $\sim 520 \mathrm{~nm}$ for the PPY thin film. Furthermore, streak camera measurement indicates the luminescence has a very long live component with a decay constant of several ns. The photo-induced triplet state absorption $\left(T_{1} \rightarrow T_{2}\right.$ transition) peaks at $\sim 600 \mathrm{~nm}$ measured by pump-probe are consistent with the radiolysis results. A triplet lifetime of $\sim 6 \mathrm{~ns}$ is measured which is again consistent with the streak camera measurement. These results lead us to believe that the long live component of the emission from PPY film is in fact phosphorescence.

\section{Acknowledgements}

This work is partially supported by the Research Grant Council of Hong Kong, EPSRC and the British Council though the UK/HK Joint Research Scheme.

\section{References}

[1] J.H. Burroughes, et al., Nature 347 (1990) 539-541.

[2] M.A. Balbo, et al., Appl. Phys. Lett. 75 (1999) 4.

[3] L.S. Swanson, J. Shinar, K. Yoshino, Phys. Rev. Lett. 65 (1990) 1140.

[4] L.S. Swanson, P.A. Lane, J. Shinar, F. Wudl, Phys. Rev. B 44 (1991) 10671.

[5] H.S. Woo, et al., Phys. Rev. B 46 (1992) 7379.

[6] X. Wei, B.C. Hess, Z.V. Vardeny, F. Wudl, Phys. Rev. Lett. 68 (1992) 666.

[7] B. Xu, S. Holdcroft, Adv. Mater. 6 (1994) 325.

[8] Yu.V. Romanovskii, et al., Phys. Rev. Lett. 84 (2000) 1027.

[9] S. Daily, et al., J. Phys: Condens. Matter 10 (1998) 5171.

[10] Y.Z. Wang, et al., Appl. Phys. Lett. 74 (1999) 3613.

[11] J.W. Blatchford, et al., Phys. Rev. B 54 (1996) 9180.

[12] T. Yamamoto, et al., J. Am. Chem. Soc. 116 (1994) 4832.

[13] A.P. Monkman, M. Halim, I.D.W. Samuel, L.E. Horsburgh, J. Chem. Phys. 109 (1998) 10372.

[14] M. Halim, et al., Synth. Met. 84 (1997) 951.

[15] A.P. Monkman, et al., Chem. Phys. Lett. 307 (1999) 303.

[16] M.E. Vaschetto, et al., J. Phys. Chem. A 103 (1999) 11096.

[17] A.P. Monkman, et al., this conference.

[18] R. Kersting, et al., Phys. Rev. Lett. 70 (1993) 3820.

[19] G.R. Hayes, et al., Phys. Rev. B 52 (1995) R11569.

[20] A.P. Monkman, et al., Soc. Plast. Eng. 57 (1999) 109.

[21] J.W. Blatchford, et al., Phys. Rev. Lett. 76 (1996) 1513.

[22] B. Kraabel, et al., Chem. Phys. 227 (1998) 83.

[23] G. Lanzani, et al., Chem. Phys. Lett. 313 (1999) 525.

[24] C. Zenz, et al., Phys. Rev. B 59 (1999) 14336.

[25] A.L. Sobolewski, et al., Chem. Phys. Lett. 180 (1991) 381.

[26] W.J. Buma, et al., J. Am. Chem. Soc. 112 (1990) 5447.

[27] G.E. Khalil, et al., Photochem. Photobiol. 28 (1978) 435. 